\title{
Fast Grid-based Path Finding for Video Games
}

\author{
William Lee and Ramon Lawrence \\ University of British Columbia
}

\begin{abstract}
Grid-based path finding is required in many video games and virtual worlds to move agents. With both map sizes and the number of agents increasing, it is important to develop path finding algorithms that are efficient in memory and time. In this work, we present an algorithm called DBA* that uses a database of pre-computed paths to reduce the time to solve search problems. When evaluated using benchmark maps from Dragon $\mathrm{Age}^{\mathrm{TM}}, \mathrm{DBA}^{*}$ requires less memory and time for search, and performs less pre-computation than comparable real-time search algorithms. Further, its suboptimality is less than $3 \%$, which is better than the PRA* implementation used in Dragon $\mathrm{Age}^{\mathrm{TM}}$.
\end{abstract}

\section{Introduction}

As games have evolved, their size and complexity has increased. The virtual worlds and maps have become larger as have the number of agents interacting. It is not uncommon for hundreds of agents to be path finding simultaneously, yet the processing time dedicated to path finding has not substantially increased. Consequently, game developers are often forced to make compromises on path finding algorithms and spend considerable time tuning and validating algorithm implementations.

Variations of $\mathrm{A}^{*}$ and PRA* are commonly used in video games [9]. The limitation of these algorithms is that they must plan a complete (but possibly abstract) path before the agent can move. Real-time algorithms such as kNN LRTA* [3] and HCDPS [8] guarantee a constant bound on planning time, but these algorithms often require a considerable amount of pre-computation time and space.

In this work, we propose a grid-based path finding algorithm called DBA* that combines the real-time constant bound on planning time enabled by using a precomputed database as in HCDPS [8] with abstraction using sectors as used in PRA* [9]. The result is a path finding algorithm that uses less space than previous real-time algorithms while providing better paths than PRA* [9]. DBA* was evaluated on Dragon Age ${ }^{\mathrm{TM}}$ maps from [10] with average suboptimality less than $3 \%$ and requiring on average less than $200 \mathrm{~KB}$ of memory and between 1 and 10 seconds for pre-computation.

\section{Background}

Grid-based path finding is an instance of a heuristic search problem. The algorithms studied in this paper, although potentially adaptable to general heuristic search, are specifically designed and optimized for 2D grid-based path finding. States are vacant 
square grid cells. Each cell is connected to four cardinally (i.e., N, E, W, S) and four diagonally neighboring cells. Out-edges of a vertex are moves available in the cell. The edge costs are 1 for cardinal moves and 1.4 for diagonal moves. Standard octile distance is used for the heuristic.

Algorithms are evaluated based on the quality of the path produced and the amount of time and memory resources consumed. Suboptimality is defined as the ratio of the path cost found by the agent to the optimal solution cost minus one and times $100 \%$. Suboptimality of $0 \%$ indicates an optimal path and suboptimality of 50\% indicates a path 1.5 times as costly as the optimal path.

An algorithm is also characterized by its response time, which is the maximum planning time per move. The overall time is the total amount of time to construct the full solution, and the average move time is the overall time divided by the number of moves made. Memory is consumed for node expansions (e.g. open and closed lists in $\mathrm{A}^{*}$ ), for storing abstraction information (e.g. regions), and for storing computed paths. Per agent memory is memory consumed for each search agent. Fixed memory is memory consumed that is shared across multiple, concurrent path finding agents.

\section{$2.1 A^{*}$}

A* [5] is a common algorithm used for video game path finding as it has good performance characteristics and is straightforward to implement. $A^{*}$ paths are always optimal as long as the heuristic function is admissible. However its overall time depends on the problem size and complexity, resulting in highly variable times. The biggest drawback is that its response time is the same as its overall time as a complete solution is required before the agent moves. A*'s memory use is variable and may be high depending on the size of its open and closed lists and the heuristic function used.

\subsection{PRA*}

The PRA* variant implemented in Dragon Age [9] was designed to improve on A* performance for path finding. Response time and per agent memory are reduced by abstracting the search space into sectors and first computing a complete solution in the abstract space. The abstraction reduces the size of the problem to be solved by $\mathrm{A}^{*}$. The abstraction requires a small amount of fixed memory. It also results in solutions that are suboptimal (between 5 to 15\%). The small trade-off in space and suboptimality is beneficial for faster response time.

\subsection{Real-time Algorithms}

There have been several real-time algorithms proposed that guarantee a constant bound on planning time per action (response time) including TBA* [1], D LRTA* [4], kNN LRTA* [3], and HCDPS [8]. TBA* is a time-sliced version of $A^{*}$ that exhibits the same properties as $\mathrm{A}^{*}$ with the added ability to control response time and per move planning time. All of the other algorithms rely on some form of pre-computation to speed up online search. 
D LRTA* [4] performs clique abstraction and generates next hop information between regions allowing an agent to know the next region to traverse to. The size of the abstraction and its pre-computation time were significant.

kNN LRTA* [3] created a database of compressed problems and online used the closest problem in the database as a solution template. Database construction time is an issue, and there is no guarantee of complete coverage. The major weakness with these two algorithms is that they fall back on LRTA* when no information is in the database, which may result in very suboptimal solutions.

HCDPS [8] performs offline abstraction by defining regions where all states are bidirectionally hill-climbable with the region representative. A compressed path database was then constructed defining paths between all region representatives. This allows all searches to be done using hill-climbing, which results in minimal per agent memory use. HCDPS is faster than PRA* with improved path suboptimality, but its abstraction consumes more memory.

\subsection{All-Pairs Shortest Path Algorithms}

LRTA* with subgoals [6] pre-computes a subgoal tree from each goal state where a subgoal is the next target state to exit a heuristic depression. Online, LRTA* will be able to use the subgoal tree to escape a heuristic depression.

It is also possible to compute a solution to the all-pairs shortest path problem and store it in a compressed form. Algorithms such as [2] store for each pair of states the next direction or state to visit along an optimal path. Depending on the compression, it is possible to achieve perfect solutions at run-time very quickly with the compromise of considerable pre-computation time and space to store the compressed databases.

\subsection{Summary}

All of these algorithm implementations balance search time versus memory used. The "best" algorithm depends on the video game path finding environment and its requirements. There are three properties that algorithms should have to be useful in practice:

- Solution consistency - The quality of the solutions (suboptimality) should not vary dramatically between problems.

- Adequate response time - The hard-limits on the response time dictated by the game must be met.

- Memory efficiency - The amount of per agent memory and fixed memory should be minimized.

The goal is to minimize suboptimality, response time, and memory usage.

\section{Approach Overview}

This work combines and extends the best features of two previous algorithms to improve these metrics. Specifically, the memory-efficient sector abstraction developed for [9] is 
integrated with the path database used by HCDPS [8] to improve suboptimality, memory usage, and response time. The DBA* algorithm performs offline pre-computation before online path finding. The offline stage abstracts the grid into sectors and regions and pre-computes a database of paths to navigate between adjacent regions. A sector is an $n \times \mathrm{x} n$ grid of cells (e.g. $16 \times 16)$. The sector number for a cell $(r, c)$ is calculated by

$$
\left\lfloor\frac{r}{n}\right\rfloor *\left\lceil\frac{w}{n}\right\rceil+\left\lfloor\frac{c}{n}\right\rfloor
$$

where $w$ is the width of the grid map. A region is a set of cells in a sector that are mutually reachable without leaving the sector. Regions are produced by performing one or more breadth-first searches until all cells in a sector are assigned to a region. A sector may have multiple regions, and a region is always in only one sector. A region center or representative state is selected for each region. The definition and construction of regions follows that in [9].

DBA* then proceeds to construct a database of optimal paths between the representatives of adjacent regions using $\mathrm{A}^{*}$. Each path found is stored in compressed format by storing a sequence of subgoals, each of which can be reached from the previous subgoal via hill-climbing. Hill-climbing compressible paths are described in [8].

To navigate between non-adjacent regions, an $R \times R$ matrix (where $R$ is the number of regions) is constructed where cell $(i, j)$ contains the next region to visit on a path from $R_{i}$ to $R_{j}$, the cost of that path, and the path itself (if the two regions are adjacent). The matrix is initialized with the optimal paths between adjacent regions, and dynamic programming is performed to determine the costs and next region to visit for all other matrix cells.

Online searches use the pre-computed database to reduce search time. Given a start cell $s$ and goal cell $g$, the sector for the start, $S_{s}$, and for the goal, $S_{g}$, are calculated using Equation 1. If a sector only has one region, then the region is known immediately. Otherwise, a BFS bounded within the sector is performed from $s$ until it encounters some region representative, $R_{s}$. This is also performed for the goal state as well to find the goal region representative, $R_{g}$.

Given the start and goal region representatives, the path matrix is used to build a path between $R_{s}$ and $R_{g}$. This path may be directly stored in the database if the regions are adjacent, or is the concatenation of paths by navigating through adjacent regions from $R_{s}$ to $R_{g}$. The complete path consists of navigating from $s$ to the region representative $R_{s}$, then following the subgoals to region representative $R_{g}$, then navigating to $g$.

The response time is almost immediate as the agent can start navigating from $s$ to the start region representative $R_{s}$ as soon as the BFS is completed. The complete path between regions can be done iteratively with the agent following the subgoals in a path from one region to the next without having to construct the entire path. The overall time and number of states expanded are reduced as the only search performed is the BFS to identify the start and goal regions if a sector contains multiple regions.

\section{Implementation Example}

We describe the implementation using a running example. 


\subsection{Offline Abstraction}

The first step in offline pre-computation is abstracting the search space into sectors as in [9]. Depending on the size of the map, the map is divided into fixed-sized sectors (e.g. $16 \times 16$ or $32 \times 32$ ). Note that there is no restriction that the sector sizes be square or a power of 2. Each sector is divided into one or more regions using BFS. The sector size serves as an upper bound for region size and limits the expansion of BFS during online path finding. If the sector size is larger, BFS within the sector will take longer. If the sector size is small, the database will be larger. In Figure 1 is a 6 sector subset of a map. The sector size is $16 \times 16$. Region representatives are shown labeled with letters. Sector 5 (middle of bottom row) has two regions $\mathrm{E}$ and $\mathrm{F}$.

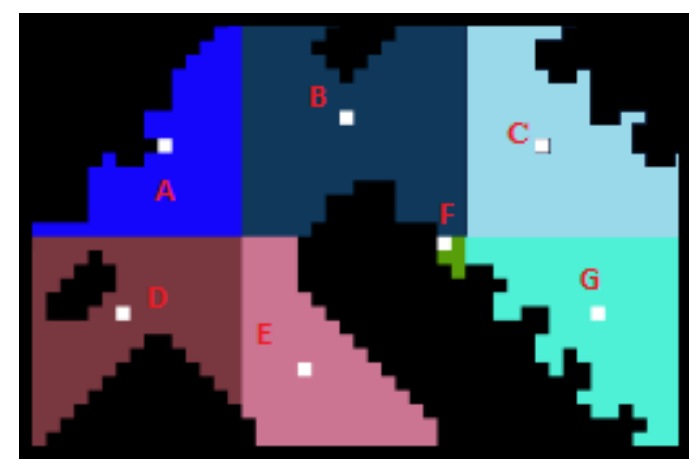

Fig. 1. Regions and Sector Abstraction Example

Region representatives are computed by summing the row (col) of each open state in the region and then dividing by the number of open states. If this technique results in a state that is a wall, adjacent cells are examined until an open state is found. DBA* and PRA* use this same technique for region representative selection in the experiments. Other methods such as proposed in [9] could also be used.

Unlike abstraction using cliques or hill-climbable regions, sector-based regions are built in $\mathrm{O}(n)$ time where $n$ is the number of grid cells. Each state is expanded only once by a single BFS. In comparison, the abstraction algorithm in HCDPS may expand a given state multiple times.

The second major advantage is that the mapping between abstract state and base state (i.e. what abstract region a given base state is in) does not need to be stored. Without compression, this mapping consumes the same space as the map. Compression can reduce the abstraction to about $10 \%$ of the map size [8].

Instead, to determine the region (and its region representative) for a given cell, first the sector is calculated. If the sector has only one region, then no search is required. Otherwise, a BFS is performed from the cell until it encounters one of the region representative states listed for the sector. This BFS is bounded by the size of the sector, and thus allows for a hard guarantee on the response time. 


\subsection{Offline Database Generation}

After abstraction, a database of paths between adjacent region representatives are computed using $\mathrm{A}^{*}$ and stored in a path database. These paths are used to populate a $R^{2}$ path matrix where $R$ is the total number of regions. Dynamic programming is performed on the matrix to compute the cost and next hop region for all pairs of regions. Note that a complete path between all pairs of regions is not stored. The path matrix only stores the cost and the next region to traverse to. Paths are only stored between adjacent regions. This is very similar to how a network routing table works where paths are outgoing links and a routing table stores the address and cost of the next hop to route a message towards a given destination.

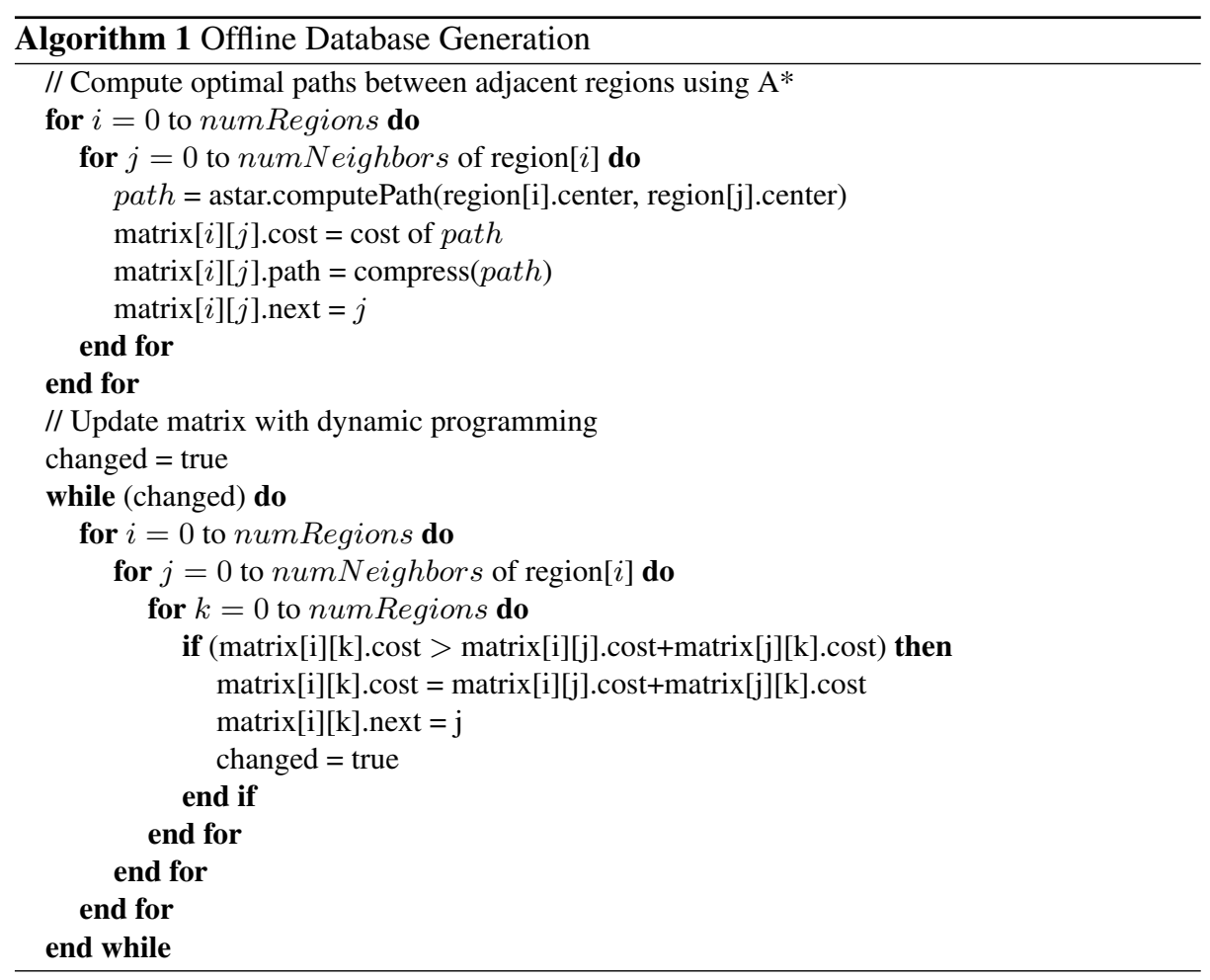

As an example, in Figure 2 is the path matrix for the 6 sector map in Figure 1. Entries in the matrix generated by dynamic programming are in italics. For example, the cost of a path from $A$ to $C$ goes through $B$ with a cost of 27.6.

\subsection{Online Path Finding}

Given a problem from start state $s$ to goal state $g$, DBA* first determines the start region and goal representatives $R_{s}$ and $R_{g}$, using BFS if multiple regions are in the sector. 


\begin{tabular}{|c|c|c|c|c|c|c|c|}
\hline & $\mathrm{A}$ & $\mathrm{B}$ & $\mathrm{C}$ & $\mathrm{D}$ & $\mathrm{E}$ & $\mathrm{F}$ & $\mathrm{G}$ \\
\hline $\mathrm{A}$ & - & $\underline{\mathrm{B}}$ & $\mathrm{B}$ & $\underline{\mathrm{D}}$ & $\underline{\mathrm{E}}$ & $B$ & $B$ \\
& & $\underline{\mathbf{1 3 . 8}}$ & 27.6 & $\underline{\mathbf{1 3 . 2}}$ & $\underline{\mathbf{2 0 . 0}}$ & 15.6 & 37.4 \\
\hline $\mathrm{B}$ & $\underline{\mathbf{A}}$ & - & $\underline{\mathbf{C}}$ & $\underline{\mathbf{D}}$ & $\underline{\mathbf{E}}$ & $\underline{\mathbf{F}}$ & $\underline{\mathbf{G}}$ \\
& $\underline{\mathbf{1 3 . 8}}$ & & $\underline{\mathbf{1 4 . 8}}$ & $\underline{\mathbf{2 1 . 6}}$ & $\underline{\mathbf{2 0 . 0}}$ & $\underline{\mathbf{1 1 . 8}}$ & $\underline{\mathbf{2 3 . 6}}$ \\
\hline $\mathrm{C}$ & $B$ & $\underline{\mathrm{B}}$ & - & $B$ & $B$ & $\underline{\mathbf{F}}$ & $\underline{\mathbf{G}}$ \\
& 27.6 & $\underline{\mathbf{1 4 . 8}}$ & & 36.4 & 34.8 & $\underline{\mathbf{9 . 8}}$ & $\underline{\mathbf{1 3 . 6}}$ \\
\hline $\mathrm{D}$ & $\underline{\mathbf{A}}$ & $\underline{\mathrm{B}}$ & $B$ & - & $\underline{\mathbf{E}}$ & $B$ & $B$ \\
& $\underline{\mathbf{1 3 . 2}}$ & $\underline{\mathbf{2 1 . 6}}$ & 36.4 & & $\underline{\mathbf{1 4 . 6}}$ & 36.4 & 45.2 \\
\hline $\mathrm{E}$ & $\underline{\mathbf{A}}$ & $\underline{\mathbf{B}}$ & $B$ & $\underline{\mathrm{D}}$ & - & $B$ & $B$ \\
& $\underline{\mathbf{2 0 . 0}}$ & $\underline{\mathbf{2 0 . 0}}$ & 34.8 & $\underline{\mathbf{1 4 . 6}}$ & & 31.8 & 43.6 \\
\hline $\mathrm{F}$ & $B$ & $\underline{\mathbf{B}}$ & $\underline{\mathrm{C}}$ & $B$ & $B$ & - & $\underline{\mathbf{G}}$ \\
& 15.6 & $\underline{\mathbf{1 1 . 8}}$ & $\underline{\mathbf{9 . 8}}$ & 36.4 & 31.8 & & $\underline{\mathbf{1 3 . 0}}$ \\
\hline $\mathrm{G}$ & $B$ & $\underline{\mathbf{B}}$ & $\underline{\mathbf{C}}$ & $B$ & $B$ & $\underline{\mathbf{F}}$ & - \\
& 37.4 & $\underline{\mathbf{2 3 . 6}}$ & $\underline{\mathbf{1 3 . 6}}$ & 45.2 & 43.6 & $\underline{\mathbf{1 3 . 0}}$ & \\
\hline
\end{tabular}

Fig. 2. Path Matrix for Example Map

In Figure 3, the start state $s$ is in region $E$, and the goal state $g$ is in region $G$. Since sector 5 contains two regions, a BFS was required to identify region $E$. Region $G$ was determined by direct lookup as there was only one region in the sector.

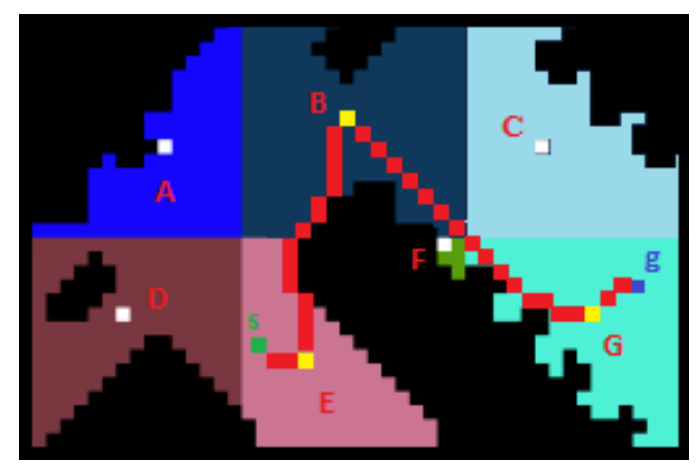

Fig. 3. Online Path Finding Example

The minimal response time possible before the algorithm can make its first move is the time for the BFS to identify the start region representative. At that point, the agent can navigate from $s$ to $R_{s}$ using the path found during BFS.

The algorithm then looks in the path matrix to find the next hop to navigate from $R_{s}$ to $R_{g}$. In this case, that is to region $B$. As these are neighbor regions, a compressed path is stored in the database, and the agent navigates using hill-climbing following its 
subgoals. Once arriving at the representative for region $B$, it then goes to $R_{g}$ (representative of region $G$ ). If a BFS path was computed to find $R_{g}$ that path can be used. Otherwise, DBA* completes the path by performing $\mathrm{A}^{*}$ bounded within the sector from $R_{g}$ to $g$. The maximum move time is the first response time for BFS. The maximum per agent memory is the number of states in a sector (as may need to search entire sector with BFS).

\subsection{Optimizations}

Several optimizations were implemented to improve algorithm performance.

Path Trimming The technique of path trimming was first described in PRA*[9] (remove last $10 \%$ of states in each concatenated path and then plan from the end of the path to the next subgoal) and extended in HCDPS (start and end optimizations). The general goal is to reduce the suboptimality when concatenating smaller paths to produce a larger path. Combining several smaller paths may produce longer paths with "bumps" compared to an optimal path. These techniques smooth the paths to make them look more appealing and have lower suboptimality.

Consider the path in Figure 3. It is visibly apparent that the agent could have navigated a better path by not navigating from $s$ to the region representative of $E$ then to the region representative of $B$. The subgoals on the path are shown, which in this case are just the region representatives themselves. Instead, the agent can perform a hill-climbing check from $s$ to the first subgoal (which is $R_{B}$ in this case) resulting in a much shorter path. A hill-climbing check if successful will shorten the path. If a hillclimbing check fails, then the agent would continue on its original planned path. Note that the agent does not actually move during the hill-climbing check. This optimization can be applied whenever there is a transition between paths put together using concatenation. The optimization is applied when navigating from $s$ to $R_{s}$, between each path fragment in the path matrix, and from $R_{g}$ to $g$. The idea is to try to go directly to the next subgoal in those cases with hill-climbing. Figure 4 shows the shorter path after applying this optimization which reduces suboptimality from $23 \%$ to $11 \%$.

Increasing Neighborhood Depth A second approach to reducing suboptimality is to increase the number of base paths computed. Instead of only pre-computing paths between adjacent regions, it is possible to increase the neighborhood depth to compute paths between regions up to $L$ steps away. This has been used in HCDPS [8] to reduce the number of path concatenations which hurts suboptimality. The tradeoff is a larger database size and longer pre-computation time. In the running example, computing paths between regions up to 2 away results in a direct path between region $E$ and region $G$ and reduces suboptimality for the example problem to $0 \%$ (when used in combination with path trimming). Increasing neighborhood depth decreases suboptimality but does not guarantee an optimal path. 


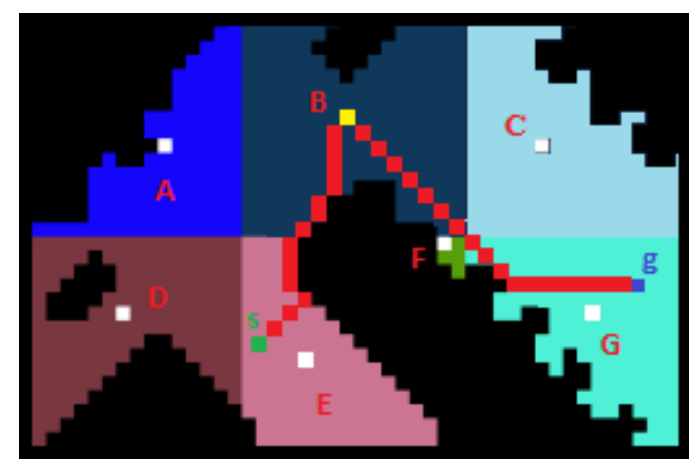

Fig. 4. Path Trimming Reduces Suboptimality When Combining Path Fragments

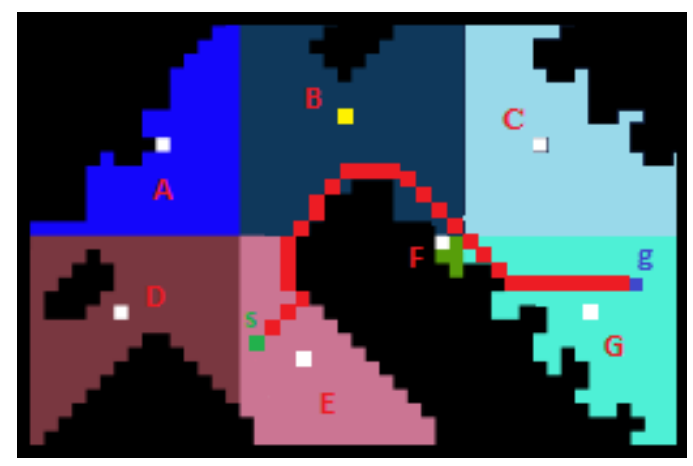

Fig. 5. Solution Path when Database Stores Paths Between Regions Up to 2 Regions Away

\section{Experimental Results}

Algorithms were evaluated on ten of the largest standard benchmark maps from Dragon Age: Origins ${ }^{\mathrm{TM}}$ available at http://movingai.com and described in [10]. The 10 maps selected were hrt000d, orz100d, orz103d, orz300d, orz700d, irz702, orz900d, ost000a, ost000t, and ost100d. These maps have an average number of open states of 96,739 and total cells of 574,132. For each map, 100 of the longest sample problems were run from the problem set.

The algorithms compared included DBA*, PRA* as implemented in Dragon Age [9], and HCDPS. HCDPS was run for neighborhood depth $L=\{1,2,3,4\}$. PRA* was run for sector sizes of $16 \times 16,32 \times 32,64 \times 64$, and $128 \times 128$ and uses the $10 \%$ path trimming and re-planning optimization. PRA* and $\mathrm{DBA}^{*}$ both did not apply region center optimization, as region representatives were computed by averaging the rows and columns of all open states in the region. DBA* was run under all combinations of neighborhood level and grid size. LRTA* with subgoals [7] was also evaluated. Algo- 
rithms were tested using Java 6 under SUSE Linux 10 on an Intel Xeon E5620 $2.4 \mathrm{GHz}$ processor with $24 \mathrm{~GB}$ of memory.

In the charts, each point in the plot represents an algorithm with a different configuration. For PRA*, there are 4 points corresponding to 16, 32, 64, and 128 sector sizes. For HCDPS there are 4 points corresponding to levels 1, 2, 3, and 4. For DBA* that combines both sets of parameters, there are separate series for each sector size $(16,32$, 64,128 ), and each series consists of 4 points corresponding to levels 1, 2, 3, and 4 .

\subsection{Online Performance}

Online performance consists of three factors: suboptimality of paths, total memory used, and average move time. Figure 6 displays suboptimality versus move time. DBA* variants (except for 128) are faster than HCDPS and PRA* and most variants have better suboptimality. DBA* has the same or better suboptimality than HCDPS for smaller sector sizes. Larger sector sizes for both DBA* and PRA* hurt suboptimality as the optimizations do not always counteract navigating through region representatives that may be off an optimal path. Larger sector sizes also dramatically increase the time for PRA* (PRA* 128 has $20 \mu s$ move time) as the amount of abstraction is reduced and the algorithm is solving a problem using $\mathrm{A}^{*}$ that is not much smaller in the abstract space. Since DBA* uses its path database rather than solving using $A^{*}$ in the abstract space, its move time does not increase as much with larger sectors. The additional time is mostly related to the BFS required to find the region representatives in the start and goal sectors. LRTA* with subgoals (shown as sgLRTA* in the legend) has different performance characteristics than the other algorithms. sgLRTA* is almost optimal. Its move time is relatively high because the subgoal trees are large, and it takes the algorithm time to identify the first subgoal to use.

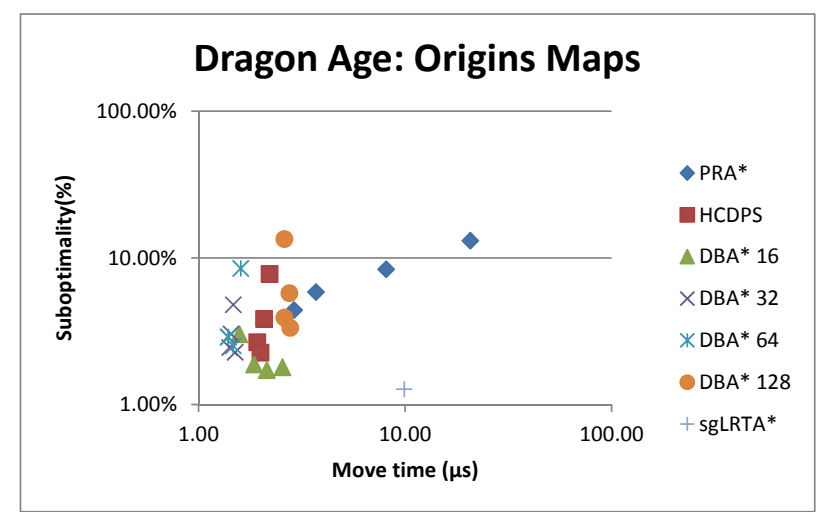

Fig. 6. Suboptimality versus Move Time 
Figure 7 compares suboptimality versus total memory used. PRA* uses less memory as storing the regions and sectors requires minimal memory. The additional memory used by DBA* to store paths between regions amounts to 50 to $250 \mathrm{~KB}$, but improves suboptimality from about $6 \%$ with PRA* to under $3 \%$. DBA* dominates HCDPS in this metric. sgLRTA* is near perfect for solution quality, but consumes significantly more memory to the point that it is not practical in this domain.

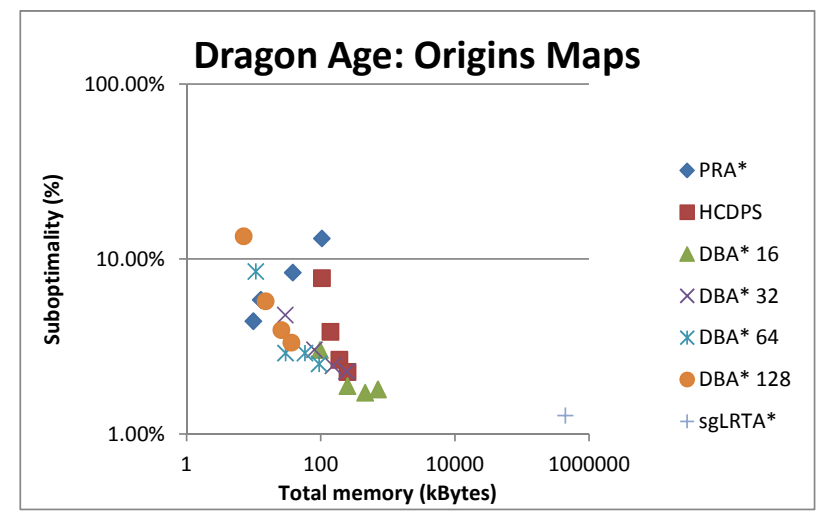

Fig. 7. Suboptimality versus Total Memory Used

For online performance, no algorithm dominates as each makes a different tradeoff on time versus space. It is arguable that the improved path suboptimality and time of $\mathrm{DBA}^{*}$ is a reasonable tradeoff for the small amount of additional memory consumed. Unlike PRA*, DBA* is optimized for static environments.

\subsection{Pre-Computation}

Pre-computation, although done offline, must also be considered in terms of time and memory required. The results are in Figure 8. PRA* consumes the least amount of memory as it only generates sectors and does not generate paths between sectors. HCDPS and DBA* both perform abstraction and path generation, although DBA* in most configurations is faster with a smaller database size. sgLRTA* that computes and compresses all paths takes considerably longer and more space than all other algorithms.

\section{Conclusions and Future Work}

Grid-based path finding must minimize response time and memory usage. DBA* has lower suboptimality than other abstraction-based approaches with a faster response time. It represents a quality balance and integration of the best features of previous algorithms. Future work includes defining techniques for efficiently updating pre-computed information to reflect grid changes. 


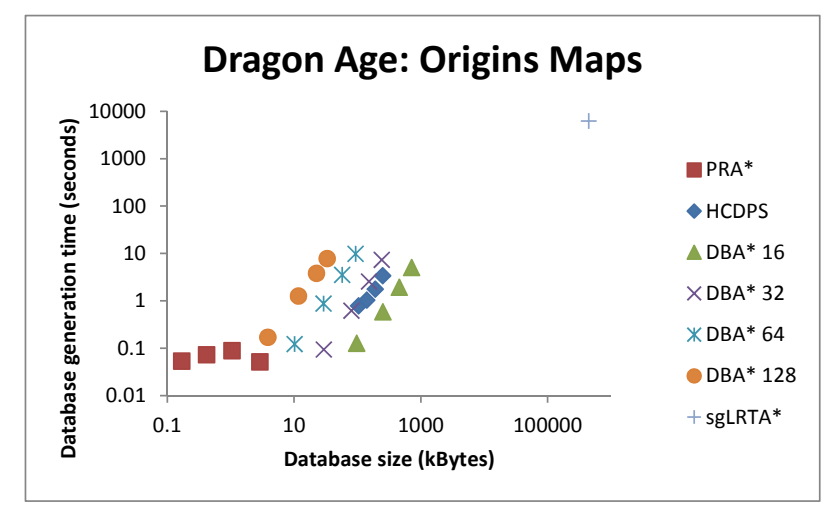

Fig. 8. Database Generation Time versus Database Generation Size

\section{References}

1. Björnsson, Y., Bulitko, V., Sturtevant, N.: TBA*: Time-bounded A*. In: Proceedings of the International Joint Conferences on Artificial Intelligence (IJCAI). pp. 431 - 436 (2009)

2. Botea, A.: Ultra-fast optimal pathfinding without runtime search. In: Proceedings of the Second Artificial Intelligence and Interactive Digital Entertainment Conference (AIIDE). pp. 122-127 (2011)

3. Bulitko, V., Björnsson, Y., Lawrence, R.: Case-based subgoaling in real-time heuristic search for video game pathfinding. Journal of Artificial Intelligence Research 39, 269-300 (2010)

4. Bulitko, V., Luštrek, M., Schaeffer, J., Björnsson, Y., Sigmundarson, S.: Dynamic control in real-time heuristic search. Journal of Artificial Intelligence Research 32, 419 - 452 (2008)

5. Hart, P., Nilsson, N., Raphael, B.: A formal basis for the heuristic determination of minimum cost paths. IEEE Transactions on Systems Science and Cybernetics 4(2), 100-107 (1968)

6. Hernández, C., Baier, J.A.: Fast subgoaling for pathfinding via real-time search. In: Bacchus, F., Domshlak, C., Edelkamp, S., Helmert, M. (eds.) Proceedings of the International Conference on Artificial Intelligence Planning Systems (ICAPS). pp. 327-330. AAAI (2011)

7. Hernández, C., Baier, J.A.: Real-time heuristic search with depression avoidance. In: Proceedings of the International Joint Conference on Artificial Intelligence (IJCAI). pp. 578583 (2011)

8. Lawrence, R., Bulitko, V.: Database-driven real-time heuristic search in video-game pathfinding. IEEE Transactions on Computer Intelligence and AI in Games PP(99) (2013)

9. Sturtevant, N.: Memory-efficient abstractions for pathfinding. In: Proceedings of Artificial Intelligence and Interactive Digital Entertainment (AIIDE). pp. 31-36 (2007)

10. Sturtevant, N.R.: Benchmarks for grid-based pathfinding. IEEE Transactions on Computer Intelligence and AI in Games 4(2), 144-148 (2012) 